\title{
The blue stragglers formed via mass transfer in old open clusters ${ }^{\star}$
}

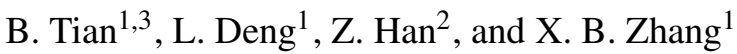 \\ 1 National Astronomical Observatories, CAS, Beijing 100012, PR China \\ e-mail: tianbin@bao.ac.cn \\ 2 National Astronomical Observatories/Yunnan Observatory, CAS, Kunming 650011, PR China \\ 3 Graduate University of Chinese Academy of Sciences, Beijing 100049, PR China
}

Received 19 January 2006 / Accepted 31 March 2006

\section{ABSTRACT}

\begin{abstract}
Aims. In this paper, we present the simulations for the primordial blue stragglers in the old open cluster M 67 based on detailed modelling of the evolutionary processes. The principal aim is to discuss the contribution of mass transfer between the components of close binaries to the blue straggler population in M 67.

Methods. First, we followed the evolution of a binary of $1.4 M_{\odot}+0.9 M_{\odot}$. The synthetic evolutionary track of the binary system revealed that a primordial blue straggler had a long lifetime in the observed blue straggler region of color-magnitude diagram. Second, a grid of models for close binary systems experiencing mass exchange were computed from 1 Gyr to 6 Gyr in order to account for primordial blue-straggler formation in a time sequence. Based on such a grid, Monte-Carlo simulations were applied for the old open cluster M 67.

Results. Adopting appropriate orbital parameters, 4 primordial blue stragglers were predicted by our simulations. This was consistent with the observational fact that only a few blue stragglers in M 67 were binaries with short orbital periods. An upper boundary of the primordial blue stragglers in the color-magnitude diagram (CMD) was defined and could be used to distinguish blue stragglers that were not formed via mass exchange. Using the grid of binary models, the orbital periods of the primordial BSs could be predicted. Conclusions. Compared with the observations, it is clear that the mechanism discussed in this work alone cannot fully predict the blue straggler population in M 67. There must be several other processes also involved in the formation of the observed blue stragglers in M 67.
\end{abstract}

Key words. stars: blue stragglers - stars: binaries: close - Galaxy: open clusters and associations: individual: M 67

\section{Introduction}

Blue stragglers (BSs) were noticed for the first time in the globular cluster (GC) M3 (Sandage 1953). These "blue extensions" lie above the main sequence turn off (MSTO) in the CMD of the GC. Afterwards, BSs have been observed in stellar systems of various types and ages. As the brightest members along the main sequence in the CMD of a given stellar system, BSs should make a considerable contribution to the blue side of the integrated light of the stellar system (Deng et al. 1999; Schiavon et al. 2004; Xin \& Deng 2005)

Observations show that BSs have higher masses than the stars at the MSTO of the host clusters (Shara et al. 1997; Nemec \& Cohen 1989). However, the classical stellar evolutionary scheme shows that single stars that are similar in mass to these BSs (more massive than MSTO) in the clusters should have already evolved away from that region in the CMD. The unusual characteristics of observed BSs cannot be explained by the theory of single-star evolution in a coeval stellar system like a cluster.

Several mechanisms for BS formation have been proposed so far, with all the established explanations related to dense environments or binary evolution, namely, direct collisions between stars, mass transfer, or coalescence in close binary systems (McCrea 1964; Strom \& Strom 1970; Hills \& Day 1976).

* Table 2 is only available in electronic form at the CDS via anonymous ftp to cdsarc.u-strasbg.fr $(130.79 .128 .5)$ or via http://cdsweb.u-strasbg.fr/cgi-bin/qcat? J/A+A/455/247
Direct physical collision hypothesis was originally presented by Hills \& Day (1976). They proposed that the remnant of a collision between two main-sequence stars could produce a blue straggler. All types of colliding encounters including singlesingle, binary-single, and binary-binary have been investigated by subsequent studies (Ouelette \& Pritchet 1998; Lombardi et al. 2002; Fregeau et al. 2004).

The proposition that BSs could be formed through mass transfer in close binary systems was presented for the first time by McCrea in 1964. He suggested that the primary could transfer material to the secondary through the inner Lagrangian point after becoming a red giant and filling up its Roche lobe. The material is stripped off of the envelope of the primary and is accommodated on the surface of the secondary. Then, as the secondary is gaining material gradually, it can became a more massive main-sequence star with a hydrogen-rich envelope. This "modified" (or, newly formed) star can stay on the upper extension of the main sequence until finishing hydrogen burning in the core. As a result of mass transfer, the star has its main-sequence lifetime doubled compared to a normal star with the same mass. Such a close binary system is called primordial BS (Davies et al. 2004). McCrea sketched such a picture and predicted that the newly formed star could be as bright as about 2.5 mag more luminous than the turn-off point of a cluster. As shown in the CMDs of star clusters, most BSs do indeed stay in that region.

The mass transfer between the components of binary systems were divided into case $\mathrm{A}$, case $\mathrm{B}$, and case $\mathrm{C}$ according to the evolutionary phases when the donor fills up its Roche 
lobe (Kippenhahn \& Weigert 1968). These three cases are associated with hydrogen burning in the core, with the rapid core contraction preceding helium ignition, and with helium having been ignited in the core.

Chen \& Han (2004) have demonstrated the possibility of BS formation via mass exchange recently. Their work reveals the evolution of both the donor and the accretor in a binary system experiencing mass transfer. They conclude that BSs may be produced in short orbital-period binaries via either the case A or case B mass-transfer scheme. They also point out that the abnormalities in the surface composition can make the accretor bluer.

A large number of BSs in various stellar systems have been observed (Mathys 1991; Landsman et al. 1997; van den Berg et al. 2001; Sandquist et al. 2003; Sandquist \& Shetrone 2003). For example, Mathys (1991) presents a detailed analysis of eleven BSs in M 67. The author points out that some of the observed BSs in M 67 show the characteristics of a binary system and of some properties of main sequence stars of the same spectral type, including surface gravities and the abundances of some chemical elements. These observational properties are verified by Milone \& Latham (Milone \& Latham 1992; Latham \& Milone 1996). Their long-term observations confirmed the binary properties of six BSs in M 67. Five out of the 6 BSs are long-period spectroscopic binaries, while the 6th (S1284) is a binary with $P=4.18 \mathrm{~d}$ and $e=0.205$. Milone \& Latham (1992) have suggested that the 6th BS is very likely a primordial BS candidate. But the eccentricity of S1284 is indeed a puzzle. The orbit of a short-period binary experiencing mass exchange should be circularized by tidal effects. Milone \& Latham (1992) mention that the eccentricity might be an artifact caused by some sort of line asymmetry in orbital solutions, an accretion disk formed during the mass transfer, or a third star in the system with a wide orbit. Among the five long-period binaries, three showed significant eccentricities, while the other two stars had nearly circular orbits. Thus Milone \& Latham concluded that there should be several different mechanisms to form these blue stragglers. When considering the observations for BSs, Leonard (1996) compared several hypotheses for the origin of blue stragglers and analyzed the observational consequences. Following these detailed comparisons, Leonard also argued that the blue straggler population in M 67 should be produced through several mechanisms.

In general, BSs in a dense stellar environment are more likely the remnants of direct collisions. In a sparse stellar environment, however, mass transfer in primordial binaries (PBs) is believed to be the primary scheme for BS formation. Ferraro et al. (2004) find that the radial distribution of the BSs in 47 Tuc appears bimodal, i.e., highly peaked in the core, decreasing at intermediate radii, and finally rising again at larger radii. Mapelli et al. (2004) studied the observed bimodal distribution of BSs in 47 Tuc with a series of simulations using a dynamical code. They find that the internal BSs mainly result from collisions and the external ones are exclusively generated by mass transfer in primordial binaries. Therefore the explanation of the observed BS distribution of 47 Tuc requires a combination of primordial BSs and collisional BSs. Piotto et al. (2004) studied nearly 3000 BSs within 56 GCs, to find that they show unexpected statistical properties - the BS population in a cluster depends neither on total mass nor on the stellar collision rate of the host cluster. Davies et al. (2004) analyzed the mechanisms of BS formation in GCs. They combined the contributions from both dynamical blue stragglers and primordial blue stragglers. The results could match the previous observation from Hubble Space Telescope (Piotto et al. 2004).
Thus Davies et al. suggest that the BSs in GCs should be formed through both collision and mass-transfer processes.

Collier \& Jenkins (1984) calculated a series of Monte-Carlo simulations for the binary system evolution of old disc clusters, the results seemed to favor the formation scheme through mass transfer in close binaries. With empirical distributions of the initial orbital parameters of close binary systems, the numbers of BSs produced in their simulations matched the observed BS number counts well.

Pols \& Marinus (1994) performed Monte-Carlo simulations of close binary evolution in young open clusters. Their simulations show that the predicted number of BSs is consistent with the observed value of the clusters younger than about $300 \mathrm{Myr}$. However, the simulations of the clusters with ages between 300 and 1500 Myr cannot produce enough BSs to fit the observations of the clusters with corresponding ages. Thus Pols \& Marinus suggest that there must be other formation processes functioning at these ages.

Hurley et al. (2001) applied their $N$-body code to model the BS population in M 67. Their code includes the cluster dynamics besides modeling the stellar and binary evolution. Compared with binary population synthesis, the $N$-body simulation can produce twice the number of BSs, which agrees with the observations. Thus Hurley et al. (2001) argue that the dynamical cluster environment of M 67 plays an important role in producing a consistent number of BSs. The authors also show that the BSs predicted by this simulation of M 67 are due to several formation processes. Later, Hurley et al. (2005) present a dynamical simulation using a direct $N$-body model for M 67. At $4 \mathrm{Gyr}$, 20 BSs are obtained, 11 of which are considered as direct mergers of two MS stars in primordial binaries. Nine of these cases were formed from Case A mass transfer, while the other two were collisions in eccentric binaries. The remaining 9 BSs were in binaries. Only two of these are primordial binaries. In their model, only 7 of 20 BSs formed from PBs were not affected by the cluster environment. Thus they suggest that the formation of the BS population in M 67 is dominated by both mass transfer and cluster dynamics.

In this paper, a detailed modelling of primordial BSs is presented. Such a treatment shows some advantages over the previous population synthesis schemes when considering nonequilibrium stars. A Monte-Carlo simulation based on these models was carried out to study the number of BSs in old open clusters from the primordial channel. In Sect. 2, we describe the evolution of primordial BSs. The results of our example model $\left(1.4 M_{\odot}+0.9 M_{\odot}\right)$ are presented. In Sect. 3, the results of Monte-Carlo simulations are presented. A summary and conclusions of our work are given in the final section.

\section{The model of primordial blue stragglers}

We adopt Eggleton's (1971, 1972, 1973) stellar evolution code to simulate the primordial BS evolution in this work. The code has been updated in subsequent work (Han et al. 1994; Pols et al. 1995, 1998) and is described by Han et al. (2000). The code uses a self-adaptive non-Lagrangian mesh and adopts Opacity Library (OPAL) radiative (Iglesias \& Rogers 1996) and molecular opacities (Alexander \& Ferguson 1994). Convective overshooting hardly affects the evolution of low-mass stars (Pols et al. 1998), so it is not considered in this work. The mass and angular momentum (AM) of the binary systems are assumed to be conservative in our calculations. 
Roche lobe overflow (RLOF) is included as a boundary condition in Eggleton's code. When RLOF takes place, the mass transfer rate is calculated via the relation

$$
\frac{\mathrm{d} m}{\mathrm{~d} t}=C \times \max \left[0,\left(\frac{R_{\mathrm{S}}}{R_{\mathrm{L}}}-1\right)^{3}\right]
$$

where $R_{\mathrm{S}}$ is the radius of the donor, and $R_{\mathrm{L}}$ that of the corresponding Roche lobe. In this work, we take $C=500 M_{\odot} \mathrm{yr}^{-1}$ to keep a steady RLOF. As discussed by Han et al. (2000), the donor overfills its Roche lobe as necessary but never overfills its lobe by much: $\left(\frac{R_{\mathrm{S}}}{R_{\mathrm{L}}}-1\right) \leq 0.001$. To avoid complications due to uncertainties in the contact systems, only the stable RLOFs are considered in this work. Our calculations are terminated once merger occurs, but the information up to the stage of contact is kept in our grids. Subsequent evolution is very important, so further study is needed.

We have neglected the influences due to tidal evolution, magnetic braking, and stellar spins on the evolution of binary systems for the following reasons. For eccentric binaries, the modelling of tidal circularization will be crucial if mass transfer happens. However, compared with adopting eccentric orbits and considering the tidal effects, adopting initially circular orbits will not affect the outcomes of a large binary population synthesis. Combining the ongoing processes of magnetic braking and tidal synchronisation will affect the mass transfer rate and merger timescale (Stepien 1995). For simplicity, the present model does not include treatment of these effects, but they will be taken up in a subsequent work.

When calculating the evolution of a donor, mass transfer rates at the corresponding ages are recorded and then used as input for adjusting the mass of the accretor, such that the evolution of the two components are synchronized. The accreted matter is assumed to be deposited onto the surface of the secondary with zero falling velocity and distributed homogeneously over the outer layers, and the evolution of the components is followed as usual for stellar evolution. Previous population synthesis studies have adopted much more simplified stellar evolution schemes. These rapid codes use analytic formulae to approximate the main characters of the stellar evolution. With them, the information on stars, such as radius, luminosity, and age, can be estimated empirically at high efficiency but at the cost of losing physical details and accuracy. Our calculations are based on realtime stellar evolution calculations. We can provide more details about the components of the binary systems, such as the information about nucleosynthesis in the core and the chemical abundance of stars.

We now present a system of $1.4 M_{\odot}+0.9 M_{\odot}$ as an example. The initial orbital separation is $5.0 R_{\odot}$ and the solar composition $[Z=0.020, Y=0.280]$ is adopted. A series of previous studies (Zahn 1966; Tassoul 1988; Goldman \& Mazeh 1991) dealt with the orbital circularization of close binaries caused by tidal interaction between the two components. It has been proved that the tidal interaction can circularize the short-period binaries, meaning that the orbital eccentricity of a close binary will decay during the course of the dynamical evolution of the system. For simplicity, we assume the orbital eccentricity $e=0$ at zero age main sequence (ZAMS) for all cases considered in this work.

The evolutionary tracks of the two components (1.4 $\left.M_{\odot}+0.9 M_{\odot}\right)$ of the example system beginning with the onset of mass transfer are given in Fig. 1. The two components of the example binary depart from the regular evolutionary tracks of single stars after mass transfer occurs at an age of 2.769 Gyr. Because the binary system begins mass transfer at

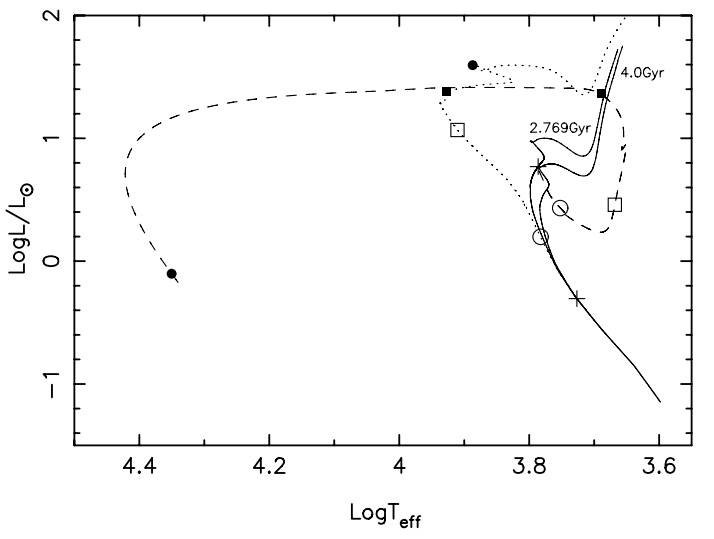

Fig. 1. The evolutionary tracks of the two components of our example binary after mass transfer begins. The dashed and dotted lines are the evolutionary tracks of the donor and the accretor. On both tracks, the crosses, open circles, open squares, filled squares, and filled circles show the positions in order: mass exchange begins, the mass ratio of the system equals 1 , the system is 4.0 Gyr old (the age when M 67 is observed), mass transfer terminates, and the core H-burning of the secondary ends. The two thin solid lines are the $2.769 \mathrm{Gyr}$ and $4.0 \mathrm{Gyr}$ theoretical isochrones.

2.769 Gyr and the age of M 67 is $4.0 \mathrm{Gyr}$, the $2.769 \mathrm{Gyr}$ and 4.0 Gyr theoretical isochrones are also plotted as a reference with solid lines in Fig. 1. When RLOF begins, the donor is still a main sequence star, so that our example binary follows the case A mass transfer scheme. The donor reaches the bottom of the giant branch after Hydrogen in its core becomes exhausted. Then the donor evolves along the giant branch. Eventually, the donor becomes a white dwarf. Due to mass accretion, the luminosity of the accretor increases. After mass transfer terminates at $4.423 \mathrm{Gyr}$, the accretor follows the evolutionary behavior of a single star again with the corresponding mass. Table 1 presents the main parameters of the binary for six key epochs in order: ZAMS, mass exchange begins, the mass ratio of the system equals 1 , the system is 4.0 Gyr old (the age when M 67 is observed), mass transfer terminates, and the core H-burning of the secondary ends.

In most cases, binary systems cannot be resolved visually in observations, so that we cannot put the components in a CMD separately. As such, we need to synthesize the total light of the system including contributions from both components to get the evolutionary track of the binary system as a whole in the CMD. In our theoretical model, we obtained the intrinsic parameters, including effective temperature, luminosity, mass, and surface gravity of the two components along their respective evolutionary tracks. Given effective temperature and surface gravity, and assuming solar abundance, we can obtain theoretical spectra (Lejeune et al. 1997, 1998) of two components along their evolutionary tracks. By convolving filter response and the corresponding spectra, the composite track of the system in the CMD can be derived as

$m=-2.5 \lg \int_{\lambda_{1}}^{\lambda_{2}} F(\lambda) \cdot \varphi(\lambda) \mathrm{d} \lambda+C$.

Where $F(\lambda)=F_{1}(\lambda)+F_{2}(\lambda)$ is the total flux of the two components, $\varphi(\lambda)$ is the response function of a filter, $\lambda_{1}$ and $\lambda_{2}$ give the range of response $\varphi(\lambda), C$ is a constant and can be determined by calibrating with observation of the Sun.

Using the response functions of the Johnson $B$ and $V$ filters, we can get the Johnson $B$ and $V$ magnitudes of the binary system. In Fig. 2, the synthetic evolutionary track of the binary system 
Table 1. Main results of the example binary $\left(1.4 M_{\odot}+0.9 M_{\odot}\right)$.

\begin{tabular}{cccccrcccc}
\hline \hline Epoch & $\begin{array}{c}\text { Age } \\
\left(10^{9} \mathrm{yr}\right)\end{array}$ & $\begin{array}{c}P \\
(\mathrm{~d})\end{array}$ & $\begin{array}{c}a \\
\left(R_{\odot}\right)\end{array}$ & $\begin{array}{c}\text { Mass } \\
\left(M_{\odot}\right)\end{array}$ & $\lg \left(L / L_{\odot}\right)$ & $\lg T_{\text {eff }}$ & $X_{\mathrm{C}}$ & $Y_{\mathrm{C}}$ & $\begin{array}{c}\dot{M} \\
\left(M_{\odot} \mathrm{yr}^{-1}\right)\end{array}$ \\
\hline 1 & 0.000 & 0.8543 & 5.0000 & 1.4000 & 0.4984 & 3.8067 & 0.700 & 0.280 & 0.0 \\
& & & & 0.9000 & -0.3802 & 3.7165 & 0.700 & 0.280 & 0.0 \\
2 & 2.769 & 0.8543 & 5.0000 & 1.4000 & 0.7307 & 3.7845 & 0.193 & 0.788 & 0.0 \\
& & & & 0.9000 & -0.3048 & 3.7266 & 0.574 & 0.406 & 0.0 \\
3 & \multirow{2}{*}{3.209} & 0.7388 & 4.5386 & 1.1500 & 0.4328 & 3.7521 & 0.096 & 0.884 & $1.62 \times 10^{-10}$ \\
& & & & 1.1500 & 0.1968 & 3.7826 & 0.532 & 0.448 & $1.62 \times 10^{-10}$ \\
4 & 4.000 & 2.1938 & \multirow{2}{*}{4.3761} & 0.5157 & 0.4589 & 3.6682 & 0.000 & 0.980 & $1.16 \times 10^{-9}$ \\
& & & & 1.7843 & 1.0680 & 3.9104 & 0.562 & 0.417 & $1.16 \times 10^{-9}$ \\
5 & 4.423 & \multirow{2}{*}{12.968} & \multirow{2}{*}{30.653} & 0.2480 & 1.3611 & 3.6891 & 0.000 & 0.000 & 0.0 \\
& & & & 2.0520 & 1.3781 & 3.9274 & 0.4000 & 0.580 & 0.0 \\
& 4.864 & \multirow{2}{*}{12.968} & \multirow{2}{*}{30.653} & 0.2480 & -0.2128 & 4.3353 & 0.000 & 0.000 & 0.0 \\
& & & & 2.0520 & 1.5954 & 3.8869 & 0.000 & 0.980 & 0.0 \\
\hline
\end{tabular}

The columns are (1) the model serial number, (2) the age, (3) the period of the binary system, (4) the separation between the two components, (5) the masses, (6) the luminosities, (7) the effective temperatures, (8) hydrogen abundance in the core, (9) helium abundance in the cores, (10) mass transfer rate.

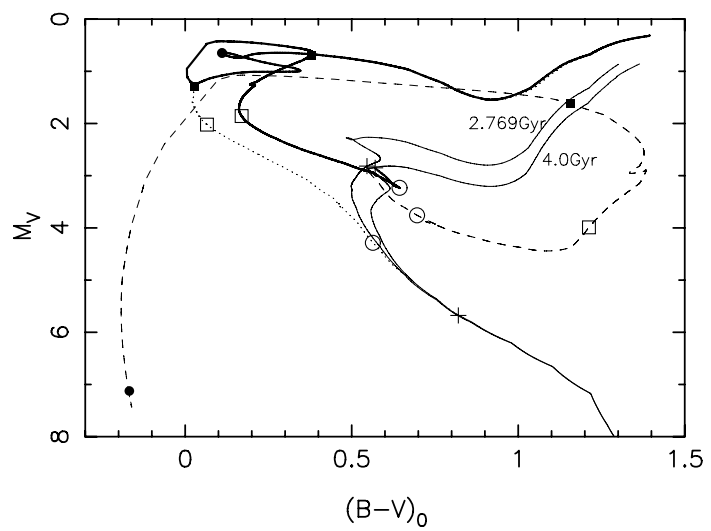

Fig. 2. The synthetic evolutionary track of the example binary system in the CMD. The thick solid line is the synthetic evolutionary track of the binary. The dashed and dotted lines are the evolutionary tracks of the donor and the accretor. The two thin solid lines are the $2.769 \mathrm{Gyr}$ and 4.0 Gyr theoretical isochrones. All the other symbols have the same meanings as in Fig. 1.

in CMD is shown. Before the mass ratio reverses, the higher luminosity of the donor dominates the synthetic evolutionary track of the binary system. After the mass ratio reverses, the accretor becomes dominant in luminosity and color. After the mass ratio becomes 1 , the binary system evolves towards the blue straggler region of the CMD. In the subsequent evolution, the binary system spends $1.228 \mathrm{Gyr}$ in the region that is bluer than MSTO of the $4.0 \mathrm{Gyr}$ isochrone until the secondary leaves the main sequence. Thus the binary system has enough lifetime in that color remaining to be observed as a BS. Evolution of the synthetic color of the example binary system is given in Fig. 3. Obviously, most of the lifetime of the binary after mass transfer starts is spent on the blue side of the CMD (defined as $(B-V)<0.545$ - the color of MSTO of the $4.0 \mathrm{Gyr}$ theoretical isochrone).

To simulate the population of the primordial BSs in old open clusters, a grid of the models of close binaries experiencing mass exchange is needed. To this aim, we calculated various combinations of binary parameters that can have mass exchange triggered for the donor masses of $0.1 M_{\odot}$ to $2.0 M_{\odot}$. The mass range of the accretor is from $0.1 M_{\odot}$ to the mass of the corresponding donor. The mass intervals of the donors and the accretors are both $0.1 M_{\odot}$. To cover all possible cases of close binaries,

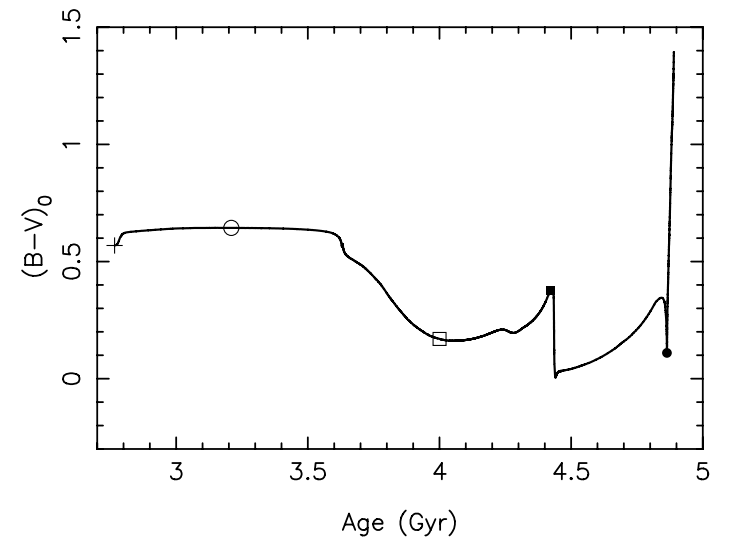

Fig. 3. Evolution of the synthetic color of the example binary system. All the symbols have the same meanings as in Fig. 1.

the orbit separation ranges from 1.0 to $50.0 R_{\odot}$ with a grid interval of $1.0 R_{\odot}$. Following the numerical scheme described above, a grid for six ages from 1.0 Gyr to $6.0 \mathrm{Gyr}$ is obtained in Table 2 (available in its electronic form at the CDS). Given the position in CMD of a primordial BS, one can estimate the parameters of the components of the binary systems with this table.

\section{Monte-Carlo simulations of the primordial BSs in $\mathrm{M} 67$}

\subsection{Initial parameters of the cluster M 67}

With the grid of primordial BS models, it is possible to statistically study the total number and distribution of primordial BSs in CMD for a given cluster. Here, we study the BSs in the old open cluster M 67 using the Monte-Carlo method. Sandquist's (2004) estimation of the age of M 67 is adopted. As claimed by Sandquist, the main features of the cluster in the CMD up to the subgiant branch can be reproduced well by a 4-Gyr isochrone, with an uncertainty lower than 0.5 Gyr. The metallicity of M 67 is believed to be solar (Hobbs \& Thorburn 1991), while Hurley et al. (2005) point out that the current total mass of M 67 is about $1400 M_{\odot}$. By considering the photometric limits in the previous work, the actual mass of M 67 ought to be slightly higher. Owing to so much detailed work on the basic parameters of the cluster and on the individual objects in the cluster including its 
BS population, models made to BSs in this cluster can be constrained well. We concentrate on the formation of primordial $\mathrm{BSs}$ in the cluster and present numerical simulations for its type. As discussed in Sect. 2, the orbital eccentricities of close binaries in our calculations are all assumed to be $e=0$. The distributions of the other initial parameters of binary systems are listed as follows:

1. Hurley et al. (2005) suggest that a system including 12000 single stars and 12000 primordial binaries at its birth can reproduce the observed parameters of M 67 in the present. Such a configuration is adopted in this work. These numbers include the stars that are gravitationally evaporated in the earlier history of M 67. Since we are considering primordial binaries that may eventually form BSs, such binary systems are presumably massive points in the cluster and therefore are unlikely to be affected by dynamical evaporation. Adopting the initial number of binaries in our case should not be very different from models including dynamical evolution. This issue will be discussed in Sect. 3.2.

2. The initial mass function (IMF) of the binary systems in a cluster has been discussed by Kroupa et al. (1991, hereafter KTG). According to the KTG IMF, the initial masses of the binary systems can be given by the generating function

$$
M(X)=0.33\left[\frac{1}{(1-X)^{0.75}+0.04(1-X)^{0.25}}-\frac{1}{1.04}(1-X)^{2}\right](3)
$$

where $M(X)$ is the binary mass in units of $M_{\odot}$, and $\mathrm{X}$ is a random number with a uniform distribution between 0 and 1 . The original single-star population in M 67 is assumed to have a mass coverage from $0.1 M_{\odot}$ to $50.0 M_{\odot}$. Thus the binary mass $M(X)$ is constrained by the limits of $0.2 M_{\odot}$ and $100.0 M_{\odot}$.

3. The uniform distribution of mass ratio assumed by Hurley et al. (2001) is adopted. The mass ratio is between $\max [0.1 /(M(X)-0.1), 0.02(M(X)-50.0)]$ and 1 .

4. The flat distribution of orbital separations from Pols \& Marinus (1994), $\Gamma(a) \propto a^{-1}$, is used here. We assume that the lower limit of the orbital separations is defined by the minimum size of the Roche lobe when RLOF takes place at ZAMS. An upper limit of 50 au as given by Hurley et al. (2005) is adopted.

\subsection{Results}

Given the initial input parameters for the cluster, KTG IMF, mass ratio, and orbit separation of the binary population at the initial stage, Monte-Carlo simulations for the M 67 BS population can be carried out. We adopt linear interpolation for the magnitudes and $B-V$ colors of all the binaries at $4.0 \mathrm{Gyr}$ using the grid listed in Table 2. The first interpolation parameter is orbital separation, the second mass ratio, and the last the mass of the donor. If the initial parameters of the binaries are not in the range of the grid, we assume that mass exchange does not occur in these binaries.

In Fig. 4 we present the results of the Monte-Carlo simulations for M 67. In our simulations, we got 19 PBs experiencing mass exchange. There are 4 in the region of BS with respect to the 4 Gyr isochrone among these PBs, which can be identified as primordial BSs. There are 4 primordial BSs shown in Fig. 4, and another 15 PBs. It also shows the observed BS sample in this cluster from Deng et al. (1999). The parameters of the 19 mass transfer PBs are listed in Table 3. According to the grid, the formation process of the mass transfer PBs is continuous during the

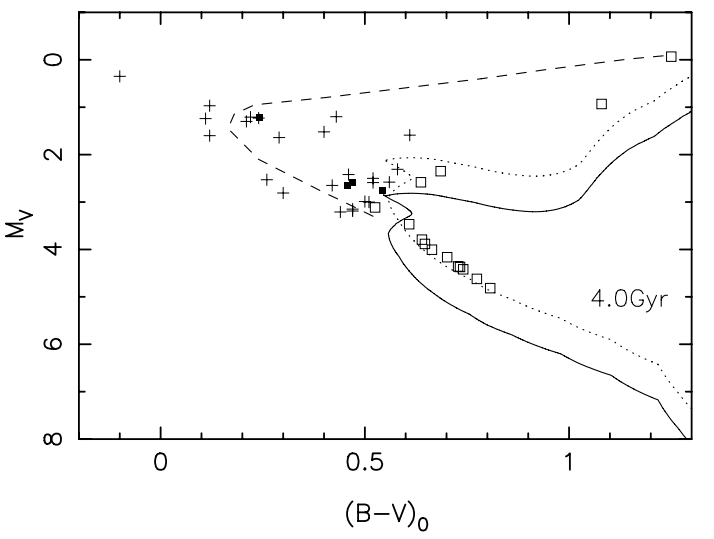

Fig. 4. The simulated CMD for the primordial BSs in M 67. The solid line is the $4.0 \mathrm{Gyr}$ theoretical isochrone. The dotted line is the equal mass photometric binary sequence. The filled squares are 4 primordial BSs. The open squares are the other 15 PBs experiencing mass exchange. The crosses are the observed BSs from Deng et al. (1999). The dashed line shows the upper edge of the simulated primordial BSs region.

Table 3. The parameters of the PBs from our Monte-Carlo simulations.

\begin{tabular}{ccccccc}
\hline \hline $\begin{array}{c}M_{1} \\
\left(M_{\odot}\right)\end{array}$ & $\begin{array}{c}q \\
\left(M_{2} / M_{1}\right)\end{array}$ & $\begin{array}{c}a \\
\left(R_{\odot}\right)\end{array}$ & $B$ & $V$ & $B-V$ & Comments \\
\hline 1.4054 & 0.8058 & 4.89 & 1.4554 & 1.2137 & 0.2417 & PBS \\
1.4775 & 0.6083 & 4.08 & 3.2915 & 2.7474 & 0.5441 & PBS \\
1.4038 & 0.5363 & 4.78 & 3.1078 & 2.6499 & 0.4579 & PBS \\
1.4713 & 0.3529 & 5.15 & 3.0676 & 2.5974 & 0.4702 & PBS \\
& & & & & & \\
1.5008 & 0.7213 & 5.20 & 1.1844 & -0.0657 & 1.2501 & DPBS \\
1.4668 & 0.7041 & 5.18 & 2.0120 & 0.9324 & 1.0796 & DPBS \\
& & & & & & \\
1.5912 & 0.4175 & 5.23 & 3.2210 & 2.5838 & 0.6372 & PPBS or PB \\
1.1887 & 0.6201 & 2.61 & 5.0869 & 4.3587 & 0.7283 & PPBS or PB \\
1.3033 & 0.9402 & 4.41 & 3.0353 & 2.3497 & 0.6856 & PPBS or PB \\
1.1998 & 0.6945 & 2.88 & 4.6690 & 4.0048 & 0.6642 & PPBS or PB \\
1.1152 & 0.9761 & 3.16 & 4.0771 & 3.4686 & 0.6085 & PPBS or PB \\
1.1954 & 0.6037 & 2.94 & 5.1030 & 4.3699 & 0.7330 & PPBS or PB \\
1.3748 & 0.5837 & 4.18 & 3.6392 & 3.1142 & 0.5250 & PPBS or PB \\
1.1952 & 0.5910 & 2.63 & 5.1598 & 4.4190 & 0.7408 & PPBS or PB \\
1.1818 & 0.5404 & 3.44 & 5.3953 & 4.6211 & 0.7742 & PPBS or PB \\
1.2600 & 0.5698 & 3.01 & 4.8657 & 4.1641 & 0.7016 & PPBS or PB \\
1.1142 & 0.8633 & 3.27 & 4.5303 & 3.8832 & 0.6471 & PPBS or PB \\
1.3256 & 0.5125 & 3.87 & 4.4328 & 3.7931 & 0.6397 & PPBS or PB \\
1.2031 & 0.4519 & 3.35 & 5.6236 & 4.8168 & 0.8069 & PPBS or PB \\
\hline
\end{tabular}

The columns are : (1) the initial mass of the donor, (2) the initial massratio of the binary, (3) the initial orbital separation between two components, (4) the Johnson $B$ magnitude, (5) the Johnson $V$ magnitude, (6) the $B-V$ color, (7) the comment on PB property: PBS-primordial blue straggler, DPBS-descendant of primordial blue straggler, PPBSprogenitor of primordial blue straggler, $\mathrm{PB}$-photometric binary.

passive evolution of the stellar population in a cluster. The binaries listed in Table 3 at 4 Gyr were produced via mass exchange in its earlier history. The 15 PBs plotted in Fig. 4 could be either progenitors or descendants of the primordial BSs. That means some of these PBs, whose mass transfer is going on and where the secondary has not yet got enough matter, might become primordial BSs later, and some were primordial BSs in an earlier epoch and have already left the region of primordial BSs in CMD at 4 Gyr. Although our model does not evolve dynamically, the BSs generated in our simulations are mostly from massive binary systems. Considering that there is a mass segregation effect 


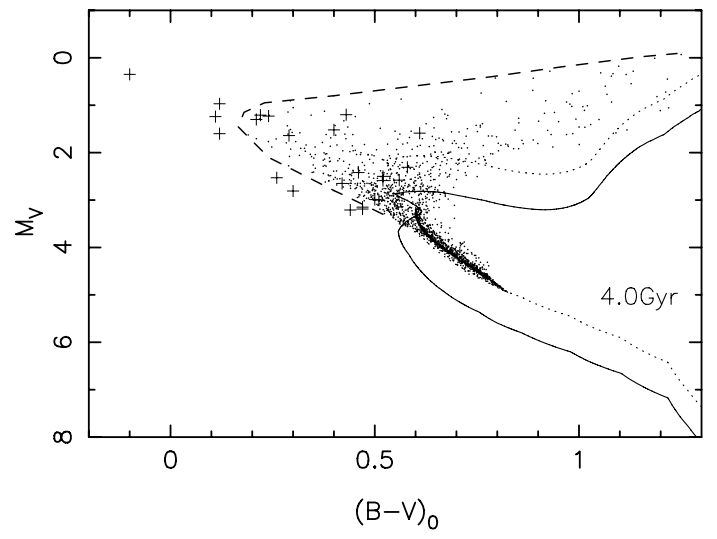

Fig. 5. The simulated CMD for the primordial BSs in a stellar system with enhanced primordial binaries. The points are the PBs experiencing mass exchange. The solid line is the $4.0 \mathrm{Gyr}$ theoretical isochrone. The dotted line is the equal mass photometric binary sequence. The crosses are the 24 photometrically selected BSs (Deng et al. 1999). The dashed line is the upper edge of the primordial BSs region.

due to the tidal force of the Galaxy, the stars lost to dynamical evaporation are mostly low mass points in the cluster, while the massive ones, however, tend to sink into the center of the cluster and will be conserved as the cluster ages. This is consistent with observational results (Mathieu \& Latham 1986; Fan et al. 1996). The progenitors of the primordial BSs are very likely the most massive stars. Although dynamical evolution, such as exchange interactions, can affect the evolution of some massive binaries, we would like to argue that the number of BSs predicted in our simulations primarily depends on the input number of the primordial binaries at zero age.

In the grid of BS models built with mass exchanging binaries, we found that there seems to be an upper limit for both coordinates of the CMD for a given age. In order to investigate such an upper boundary, we followed the same simulation method as above, but augmenting the total number of primordial binaries, thereby raising the total number of binaries that enter BS region in the case of M 67. The simulationin in which the number of primodial binaries has been raised $(N=1200000)$ is shown in Fig. 5, and 1984 PBs experiencing mass transfer were obtained. By counting the number of the binaries staying in BS region, we find about $11 \%$ primordial BSs among 1984 PBs. Most of these PBs stay in between the turn off and about 2 mag below the turn off of the $4.0 \mathrm{Gyr}$ theoretical isochrone. In our simulations for M 67, there are $11 \mathrm{PBs}$ remaining in that region. The observed CMD (Fan et al. 1996) shows that quite a few stars still remain in such a region, the characteristics of these stars are still uncertain, most of them should be photometric binaries, and some of these stars could be the low mass primordial binaries that also experienced mass exchange.

An empirical upper limit for the primordial BSs can be drawn from the simulation (Fig. 5). This upper limit in both luminosity and temperature are defined by the upper profile of the results, also visible in Fig. 4. We can define a primordial $\mathrm{BS}$ region that is confined between this upper limit and the given isochrone. In other old open clusters, we can also define such a primordial BS region. The observed short period binaries staying in the region should be primordial BSs. According to our grid of the models of those close binaries experiencing mass exchange, the blue edge of the primordial BS region shifts redwards following the evolution of a cluster. The blue edge shifts very slowly near $(B-V)=0.1$ from $1.0 \mathrm{Gyr}$ to $4.0 \mathrm{Gyr}$, and
Table 4. The parameters of 24 observed BSs in M 67.

\begin{tabular}{ccccc}
\hline \hline Sanders number & $m_{\mathrm{V}}$ & $B-V$ & $P(\mathrm{~d})$ & Eccentricity \\
\hline S0977 & 10.04 & -0.10 & & \\
S1434 & 10.66 & 0.12 & & \\
S1066 & 10.93 & 0.11 & & \\
S1267 & 10.90 & 0.22 & 846 & 0.475 \\
S1284 & 10.92 & 0.24 & 4.18 & 0.205 \\
S1263 & 10.99 & 0.21 & & \\
S0968 & 11.29 & 0.12 & & \\
S0975 & 10.89 & 0.43 & 1221 & 0.088 \\
S1082 & 11.21 & 0.40 & $1.068+1189$ & 0.57 \\
S0752 & 11.33 & 0.29 & 1003 & 0.317 \\
S1072 & 11.28 & 0.61 & & \\
S1280 & 12.22 & 0.26 & & \\
S0997 & 12.11 & 0.46 & 4913 & 0.342 \\
S1195 & 12.34 & 0.42 & 1154 & 0.066 \\
S0792 & 12.00 & 0.58 & & \\
S0277 & 12.28 & 0.52 & & \\
S2226 & 12.50 & 0.30 & & \\
S1273 & 12.27 & 0.56 & & \\
S0984 & 12.19 & 0.52 & & \\
S1005 & 12.68 & 0.50 & & \\
S0751 & 12.69 & 0.51 & & \\
S1036 & 12.84 & 0.47 & & \\
S0145 & 12.90 & 0.44 & & \\
S2204 & 12.88 & 0.47 & & \\
\hline
\end{tabular}

reaches the bluest color of $(B-V)=0.03$ at $4.6 \mathrm{Gyr}$. The data of the binary systems at $4.6 \mathrm{Gyr}$ are from our detailed modelling of the primordial BSs. After $4.6 \mathrm{Gyr}$, the blue edge moves to the red side of the CMD and reaches the position $(B-V)=0.36$ at 6.0 Gyr.

A sample of 24 photometrically selected BSs (Deng et al. 1999) are plotted in Fig. 5, and their parameters are listed in Table 4. The orbital parameters of 7 stars have already been measured (Milone \& Latham 1992; Latham \& Milone 1996; Sandquist et al. 2003), and S1284 has been shown to have a short orbital period $(P=4.18 \mathrm{~d})$. Chen \& Han (2004) proved that $\mathrm{S} 1284$ could have been formed via mass exchange. But they also point out that the non-zero eccentricity of S1284 is a puzzle, because tidal effects would circularize the orbit of a binary with a short orbital period in the mass transfer scheme. This problem was discussed by Milone \& Latham (1992) who mention that the eccentricity might not be real, might be affected by an accretion disc, or a distant third star in a wide orbit. Sandquist et al. (2003) point out that $\mathrm{S} 1082$ should be a triple system made up of a close binary $(P=1.068 \mathrm{~d})$ and another star. The eccentricity of S1082 in Table 4 is for the orbit of the third star. The corresponding period is $1189 \mathrm{~d}$, which is too long to have any important dynamical effects on the close binary system. The primary of the close binary in S1082 is a BS. S1284 and the close binary in S1082 are the only BSs in close binary systems that can be accounted for by mass exchange mechanism.

The period distribution of the grid of primordial BSs at 4.0 Gyr is given in Fig. 6. The grid of the binaries seems to have two separate branches, one below 5 days and the other over 7 days. The first branch shows an obvious trend; i.e. the bluer the color of the system, the longer the period. The other one is likely to behave similarly but have a different slope and some offsets in period. As the grid is not fine enough for the second branch in this work, no details can be given at present. Work on this point is in progress and will be presented in a future paper. However, the 2 observed primordial BSs with short periods S1284 and S1082 are already well confined in the current grid, as clearly shown 


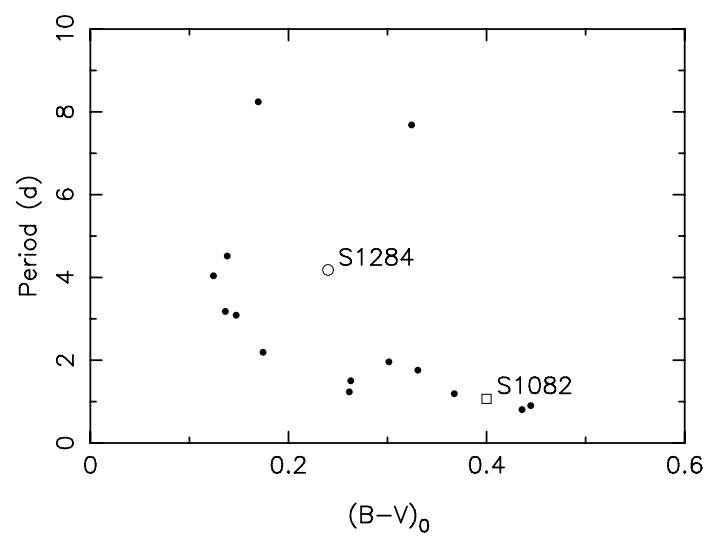

Fig. 6. The period distribution of the grid of primordial BSs at 4.0 Gyr. The open circle and square show the positions of S1284 and S1082.

in Fig. 6, where the periods of the two observed BSs match the trend of the first branch closely.

Most of the observed BSs in binary systems show high eccentricity. This contradicts the assumption $(e=0)$ in our model. But we cannot confirm that this high eccentricity is the initial nature of these binaries. Hurley et al. (2005) suggest that dynamical encounters within the cluster environment and perturbations from nearby stars or binaries could alter the orbit parameters of the primordial binaries. Nonetheless, we point out that some of these BSs with high eccentricity might have been created via wind accretion in wide binaries. In that case, the orbits can be wide enough to remain eccentric.

There are 9 observed BSs that stay above the upper limit defined above. We tend to conclude that these stars should not be the primordial BSs, so that the true nature of these BSs should be understood by other formation mechanisms. However, as the blue and upper boundaries of the primordial BS region changes with the age of a cluster, our conclusion that the BSs are above the limit is not exclusive. For instance, the 9 stars would be covered by the primordial BS region, if the real age of M 67 were around 4.5 Gyr. Considering the distribution of orbital inclinations and the current number of BSs in short period binary systems, the ratio of primordial BSs in M 67 is low, which agrees with the predicted number of BSs in our simulations.

\section{Summary and conclusions}

According to numerous observations of BSs in various stellar systems, BSs in a given stellar system should be formed via different formation mechanisms (Piotto et al. 2004). Generally, direct collisions are considered as the principal mechanism to form BSs in a dense stellar environment (Fregeau et al. 2004), while mass transfer in a close binary system is regarded as the main way to form BSs in a sparse environment (Mathys 1991). Based on the scheme outlined long ago by McCrea (1964), we have presented detailed modelling of PBs experiencing mass exchange and simulated the situation of BSs in the old open cluster M 67.

The evolutionary model of BS formed via mass transfer is shown in this work. As previous studies have been based on simplified evolution schemes, detailed modelling of masstransfer binaries may have positive effects on population synthesis and reveal more information on these binaries. A binary of $1.4 M_{\odot}+0.9 M_{\odot}$ was set as an example. In this example, both the donor and the accretor follow idiosyncratic evolutionary schemes that deviate completely from the classical evolution scenario of single stars. By summing up the total light of the two components in the example binary system along their individual evolutionary tracks, we get a synthetic evolutionary track of the example binary system in the CMD. According to the synthetic track, the binary spends $1.228 \mathrm{Gyr}$ in the region where it is bluer than the MSTO of the $4.0 \mathrm{Gyr}$ isochrone until the secondary leaves the main sequence. Thus the example binary lasts long enough in the target region to be observed as a BS.

A grid of primordial BS models for old stellar populations from 1.0 Gyr to 6.0 Gyr is also given. Using this grid, we can understand the BS formed in this channel and simulate primordial BSs in real star clusters in these ages. According to the grid, the formation process of the PBs is continuous during the evolution of a cluster-like M 67. The PBs simulated by our calculation at a certain epoch were produced via mass exchange, which happened before the time of observations. At this epoch, a star whose mass is lower than the one at the MSTO of the corresponding isochrone may evolve into a straggling phase with respect to a single-star scenario by gaining material from the donor, which brings the whole binary system into a peculiar evolutionary scheme.

Based on detailed modelling of primordial BSs, a Monte-Carlo simulation of the old open cluster M 67 was made. The simulation defines an upper boundary of primordial BS in the CMD. Thus we can define a primordial BS region between this upper limit and the given isochrone. This region can better constrain the position of the BSs formed via mass transfer in the CMD, which is helpful for distinguishing the formation channels of the observed BSs. We would like to argue that the BSs with short periods entering the primordial BS region should have a mass-transfer origin. Our model only predicts 4 primordial BSs (1/6 of the observed number count). For the case of M 67, there are two such instances (S1284 and the close binary component of the triple system S1082). The observation fairly agrees with our prediction for the distribution of orbital inclinations of binary systems.

Hurley et al. (2001) tested different combinations of these initial parameter distributions in their binary population synthesis. The maximum number of blue stragglers generated in their simulations is 8.2 per 5000 binaries at $4.2 \mathrm{Gyr}$, while the minimum is 1.1 out of 5000. Obviously, these results cannot account for the number of observed BSs in M 67. Thus they conclude that cluster dynamics is beneficial not only for explaining BSs with eccentric and/or wide orbit but also for increasing the simulated BSs number. In their dynamical simulations, 22 BSs are generated, but only one of them is in a binary, while the predicted number of BSs reaches the maximum at $3.653 \mathrm{Gyr}$ with seven of BSs in binaries. As a result, they thought that the blue stragglers observed in M 67 should be formed through several processes. Recently, Hurley et al. (2005) presented a new result of dynamical simulation using a direct $N$-body model and also performed new BS population synthesis for M 67. At 4 Gyr, the new dynamical model generates 20 blue stragglers with 9 of them in binaries, while BS population syntheses produce $25 \mathrm{BSs}$ with 6 in binaries. From both studies, it is concluded that dynamical processes destroy BSs as well as creating new ones. According to their new results, Hurley et al. (2005) argue that both the primordial binary population and the dynamical environment play essential roles in generating BSs in open clusters. There is an obvious difference between their results of population synthesis and our simulations. The primary reason is that we are strictly limited to PBs from case A. Instead, they have implemented many more BS formation channels in their population synthesis, including Case B and C mass transfer schemes and wind 
accretion. After all, inclusion of tides and a distribution of eccentricities could also affect the number of BSs produced via the Case A scheme.

With the mechanism of primordial BS formation, the period distribution of the PBs can be predicted. This is proved by a comparison between the 2 observed BSs with short periods in M 67 and our theoretical results. This is a preliminary approach to this observable property of primordial BSs, so more studies and finer grid of binary models are needed.

In fact, there are some primordial BS formation processes that are not considered in the present work, such as the merger of short period binaries and wind accretion in wide binaries, the latter case can possibly account for the observed BSs in wide binaries. Even within the primordial BS region, other mechanisms are also needed. More observations, especially of binaries and orbital parameters, are needed in order to understand the BS population in M 67.

Acknowledgements. We would like to thank the National Science Foundation of China (NSFC) for support through grants 10333060, 10573022, and 10521001. We are grateful to the referee for his/her useful and inspirational comments that helped to improve this work.

\section{References}

Alexander, D. R., \& Ferguson, J. W. 1994, ApJ, 437, 879

Chen, X. F., \& Han, Z. W. 2004, MNRAS, 355, 1182

Collier, A. C., \& Jenkins, C. R. 1984, MNRAS, 211, 391

Davies, M. B., Piotto, G., \& De Angeli, F. 2004, MNRAS, 348, 129

Deng, L., Chen, R., Liu, X. S., \& Chen, J. S. 1999, ApJ, 524, 824

Eggleton, P. P. 1971, MNRAS, 151, 351

Eggleton, P. P. 1972, MNRAS, 156, 361

Eggleton, P. P. 1973, MNRAS, 163, 279

Fan, X. H., Burstein, D., Chen, J. S., et al. 1996, AJ, 112, 628

Ferraro, F. R., Beccari, G., Rood, R. T., et al. 2004, ApJ, 603, 127

Fregeau, J. M., Cheung, P., Portegies Zwart, S. F., \& Rasio, F. A. 2004, MNRAS, 352, 1

Goldman, I., \& Mazeh, T. 1991, ApJ, 376, 260

Han, Z., Podsiadlowski, Ph., \& Eggleton, P. P. 1994, MNRAS, 270, 121

Han, Z., Tout, C. A., \& Eggleton, P. P. 2000, MNRAS, 319, 215
Hills, J., \& Day, C. 1976, Astro. Lett., 17, 87

Hobbs, L. M., \& Thorburn, J. A. 1991, AJ, 102, 1070

Hurly, J. R., Tout, C. A., Aarseth, S. J., \& Pols, O. R. 2001, MNRAS, 323, 630

Hurly, J. R., Pols, O. R., Aarseth, S. J., \& Tout, C. A. 2005, MNRAS, 363, 293

Iglesias, C. A., \& Rogers, F. J. 1996, ApJ, 464, 943

Kippenhahn, R., \& Weigert, A. 1968, Z. Astrophys., 65, 251

Kroupa, P., Tout, C. A., \& Gilmore, G. 1991, MNRAS, 251, 293

Landsman, W., Aparicio, J., Bergeron, P., Di Stefano, R., \& Stecher T. P. 1997, ApJ, 481, 93

Latham, D. W., \& Milone, A. A. E. 1996, in The Origins, Evolutions, and Destinies of Binary Stars in Clusters, ed. E. F. Milone, \& J. C. Mermilliod ASP Conf. Ser. 90, 385

Lejeune, Th., Cuisinier, F., \& Buser, R. 1997, A\&AS, 125, 229

Lejeune, Th., Cuisinier, F., \& Buser, R. 1998, A\&AS, 130, 65

Leonard, P. J. T. 1996, ApJ, 470, 521

Lombardi, J. C., Warren, J. S., Rasio, F. A., Sills, A., \& Warren, A. R. 2002, ApJ, 568,939

Mapelli, M., Sigurdsson, S., Colpi, M., et al. 2004, ApJ, 605, L29

Mathieu, R. D., \& Latham, D. W. 1986, AJ, 92, 1364

Mathys, G. 1991, A\&A, 245, 467

McCrea, W. H. 1964, MNRAS, 128, 147

Milone, A. A. E., \& Latham, D. W. 1992, in Evolutionary Processes in Interacting Binary Stars, ed. Y. Kondo, R. F. Sisteró, \& R. S. Polidan, IAU Symp. 151, 475

Nemec, J. M., \& Cohen, J. G. 1989, ApJ, 336, 780

Ouellette, J. A., \& Pritchet, C. J. 1998, AJ, 115, 2539

Piotto, G., De Angeli, F., King, I. R., et al. 2004, ApJ, 604, 109

Pols, O. R., \& Marinus, M. 1994, A\&A, 288, 475

Pols, O. R., Tout, C. A., Eggleton, P. P., \& Han, Z. 1995, MNRAS, 274, 964

Pols, O. R., Schroder, K.-P., Hurley, J. R., Tout, C. A., \& Eggleton, P. P. 1998, MNRAS, 298, 525

Sandage, A. R. 1953, AJ, 58, 61

Sandquist, E. L. 2004, MNRAS, 347, 101

Sandquist, E. L., \& Shetrone, M. D. 2003, AJ, 126, 2954

Sandquist, E. L., Latham, D. W., Shetrone, M. D., \& Milone, A. A. E. 2003, AJ, 125,810

Schiavon, R. P., Caldwell, N., \& Rose, J. A. 2004, AJ, 127, 1513

Shara, M. M., Saffer, R. A., \& Livio, M. 1997, ApJ, 489, 59

Stepien, K. 1995, MNRAS, 274, 1019

Strom, S. E., \& Strom, K. M. 1970, ApJ, 162, 523

Tassoul, J. L. 1988, ApJ, 324, 71

van den Berg, M., Orosz, J., Verbunt, F., \& Stassun, K. 2001, A\&A, 375, 375

Xin, Y., \& Deng, L. ApJ, 2005, 619, 824

Zahn, J. P. 1966, AnAp, 29, 489 